\title{
[010] Oxygen Ordering at Grain Boundaries in $\mathbf{M g B}_{2}$
}

\author{
J. C. Idrobo ${ }^{*}$, R. F. Klie*, and N. D. Browning* , A.C. Serquis ${ }^{* *}$, F.M. Mueller ${ }^{* *}$ \\ *Dept. of Physics, University of Illinois at Chicago, MC 273, Chicago, IL 60607, USA \\ ** Superconductivity Technology Center, Los Alamos National Laboratory, Los Alamos NM 87545
}

The discovery of superconductivity in $\mathrm{MgB}_{2}$, with a transition temperature of $\mathrm{T}_{\mathrm{c}}=39 \mathrm{~K}$ [1] has focused scientific attention towards an understanding of its superconducting properties. One interesting observation that is related to both the mechanical properties and to flux pinning, involves oxygen segregation to grain boundaries [2] and the formation of oxygen precipitates in the bulk of polycrystalline materials [3]. In this study, we elaborate on the initial observation of oxygen segregation/precipitation and show through experimental Z-contrast images, electron energy loss spectroscopy (EELS) and simulations, that ordered $\mathrm{MgB}_{\mathrm{x}} \mathrm{O}_{\mathrm{y}}$ phases form in these segregated/precipitated regions.

The Z-contrast images and EELS were obtained from the $200 \mathrm{kV}$ JEOL 2010F STEM at UIC, which has an objective aperture $\sim 15 \mathrm{mrad}$, a point resolution of $\sim 0.14 \mathrm{~nm}$ in scanning mode, and an angular range of 0-52 mrad for EELS and 52-200 mrad for the ADF detector. Under these conditions, the ADF detector allows the direct observation of the structure of the grains, and the image can be used to position the electron probe for EELS. The spatial resolution of the spectra is slightly degraded compared to the images, due to the localization of the signal and probe tails. However, the compositional and bonding information is sufficiently localized that it can be correlated with specific atomic locations in the image. Figure 1 shows the experimental Z-contrast image obtained from an oxygen rich grain boundary. Increased intensity in every second plane implies the presence of oxygen ordering (higher $\mathrm{Z}$ compared to Boron). This ordering is confirmed by the $\mathrm{O}-\mathrm{K}$ edge spectra shown in figure 1. Quantification of these spectra indicates an oxygen doping level of $25 \%$ [3] and this is the concentration used for the simulations.

To confirm that these contrast variations are caused by oxygen ordering, Z-contrast image simulations were performed using the program developed by Kirkland [4]. To generate the images, the program assumes that the beam in the microscope interacts with an effective potential of the specimen as a whole. Here the effective potential of $\mathrm{MgB}_{2}$ is assumed to be a linear superposition of the potentials for each atom, and this is calculated with a relativistic form of the Hartree-Fock [4] method. The algorithm used by the program to generate a Z-contrast image does the following: A focused probe is calculated by taking into consideration aberrations over the objective aperture, then the electron probe passes through the $\mathrm{MgB}_{2}$ and is modulated by the transmission function. The Fourier Transform of the transmitted wave function is projected onto the annular dark field (ADF) detector. The square modulus of this wave function in the ADF detector is integrated over the entire scattered angle and the result is processed (with the addition of noise) to obtain a final simulated image that can be compared with experiment. Figure 2 shows the simulated images of $\mathrm{MgB}_{2}$ in the [010] orientation without oxygen and with oxygen respectively. These results confirm the presence of oxygen ordering in the oxygen rich phase. The effect of this ordered structure on the transport properties will be the subject of future research [5]. 
References:

[1] Nagamatsu J., Nakawa N. et al. (2001) Nature (London) 410,63.

[2] Klie R. F., Idrobo J. C., and Browning N. D. Reagan K.A., Rogado N.S. and Cava R.J. coauthors. (2001) Applied Physics Letters. 79(12).

[3] Klie R. F., Idrobo J. C., and Browning N. D. Serquis A.C . and Mueller F.M. (2002) submitted Applied Physics Letters.

[4] Kirkland E. J. (1998) Advanced Computing in Electron Microscopy. New York, Plenum Press.

[5] This work is supported by the U.S. Department of Energy under Grant No. DOE FG02 96ER45610.

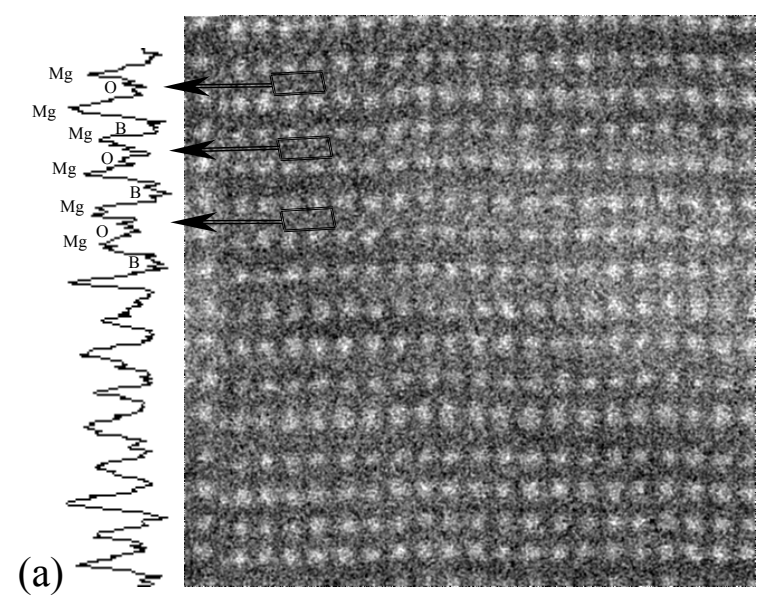

(b)

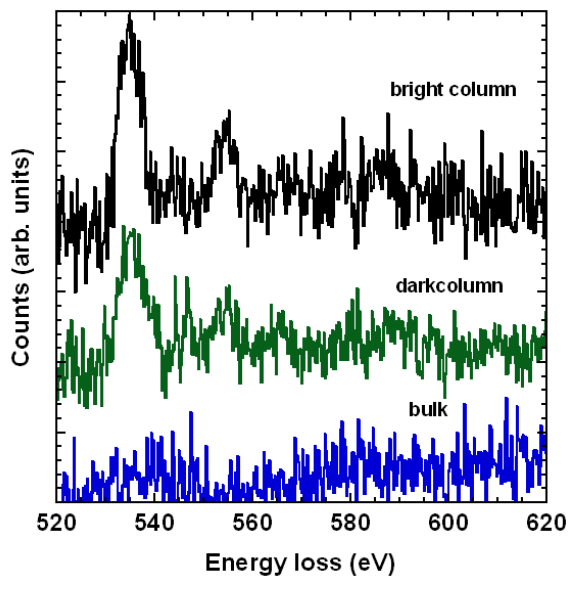

Figure 1: (a) Z-contrast image of $\mathrm{MgB}_{2}$ in the [010] orientation with oxygen ordering and an intensity profile. (b) $\mathrm{O}-\mathrm{K}$ edges from the bright and dark columns, concentration of $25 \%$ of oxygen was found in the bright columns.

(a)

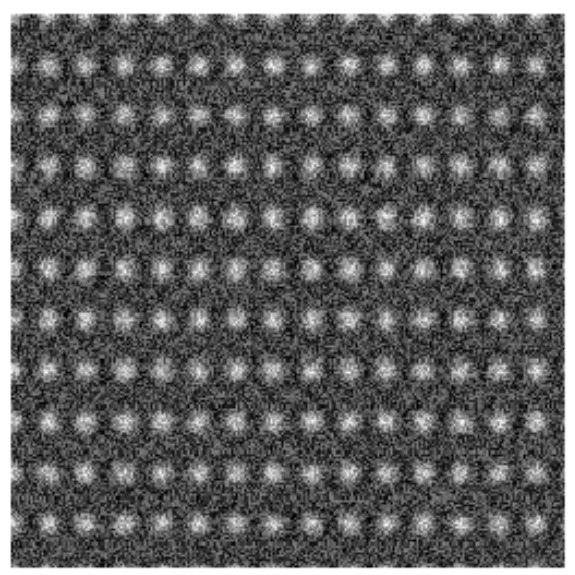

(b)

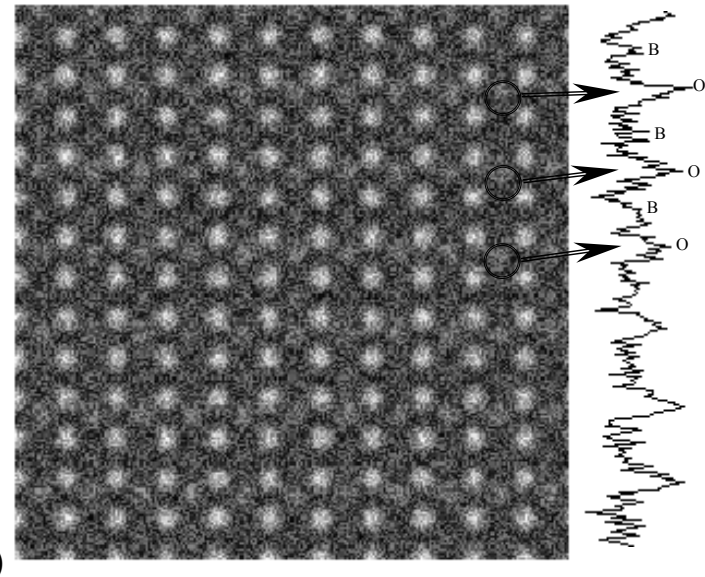

Figure 2: Simulated Z-contrast image of $\mathrm{MgB}_{2}$ in the [010] orientation, (a) without oxygen ordering, and (b) with $25 \%$ oxygen ordering. 